\title{
COMMENTARY
}

\section{Genomic Catastrophe and Neoplastic Transformation}

\author{
William B. Coleman
}

From the Department of Pathology and Laboratory Medicine, Curriculum in Toxicology, UNC Program in Translational Medicine, UNC Lineberger Comprehensive Cancer Center, University of North Carolina School of Medicine, Chapel Hill, North Carolina

\section{Cell Fusion and Cancer}

Theodor Boveri published his groundbreaking work approximately 100 years ago suggesting that neoplastic transformation and cancer induction was "...a consequence of a certain abnormal chromosome constitution...", p21 This observation was particularly insightful given that it came decades before the general acceptance of DNA as the genetic material in animal cells, and well before the molecular underpinnings of cancer were defined.

We have witnessed a molecular revolution in biology and have gained great understanding of the genes, pathways, and molecular mechanisms of cancer development and progression. ${ }^{2}$ Despite our increased understanding of the hallmark features of cancer ${ }^{3}$ and the governing mechanisms, we lack fundamental understanding of how cancer develops (spontaneously) from normal cells in the absence of inherited gene defects, infection by oncogenic viruses, or exposure to environmental carcinogens. The study by Zhou et $\mathrm{al}^{4}$ used an innovative experimental design that enabled examination of oncogenic molecular events occurring early after fusion of normal (nonneoplastic) rat intestinal crypt epithelial (IEC-6) cells. The objective of this study was to determine whether a cell fusion event involving normal cells will precipitate molecular alterations that drive neoplastic transformation and tumorigenesis. Cell fusion has been suggested as a possible initiating event in cancer development based on several observations: i) cell fusion events can be detected in existing cancers, ${ }^{5}$ ii) cell fusion is associated with genomic instability, which could drive neoplastic transformation, and iii) cell fusion may account for neoplastic transformation among nonproliferative differentiated cell types. ${ }^{7}$

\section{Experimental Model of Cell Fusion}

Zhou et al $^{4}$ used rat IEC- 6 cells ${ }^{8}$ which have a stable diploid karyotype, lack cellular characteristics of neoplasticallytransformed cells in vitro, and fail to form tumors in animals after repeated passaging in cell culture. IEC-6 cells were fluorescently labeled using either green (CSFE) or red (SNARF-1) dyes, mixed, cell fusion was mediated with $50 \%$ polyethylene glycol (PEG), and the resulting cell population was subjected to fluorescence-activated cell sorting (FACS) to identify fused and non-fused cells. Fused cells were larger in size and exhibited dual fluorescent signal, whereas non-fused cells were of normal size and displayed only one fluorescent marker. ${ }^{4}$ Fused cells were sorted and clonal populations were established and subsequently characterized for growth properties in vitro, markers of DNA damage, and tumorigenic potential in vivo. ${ }^{4}$

\section{Cell Fusion Engenders Genomic Instability and DNA Damage}

Fusion-derived IEC-6 cell clones were successfully established from $19 \%$ of FACS-sorted cells, ${ }^{4}$ suggesting that a subset of cell fusion events result in clones that can proliferate and expand. Among the fusion-derived IEC-6 cell clones examined, $41 \%$ were aneuploid and $56 \%$ were near-diploid, whereas $86 \%$ of non-fused cell clones were diploid. ${ }^{4}$ This suggests that cell fusion engenders genomic instability.

\footnotetext{
Accepted for publication May 7, 2015.

Disclosures: None declared.

Address correspondence to William B. Coleman, Ph.D., Department of Pathology and Laboratory Medicine, CB\#7525, Room 515 BrinkhousBullitt Bldg., University of North Carolina School of Medicine, Chapel Hill, NC 27599. E-mail: wbcolemn@med.unc.edu.
} 

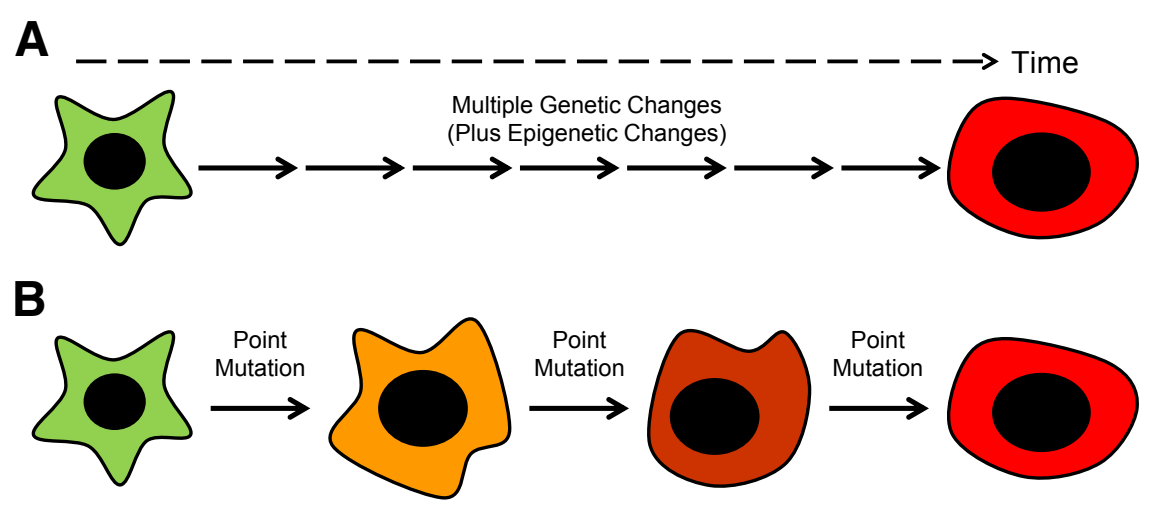

c

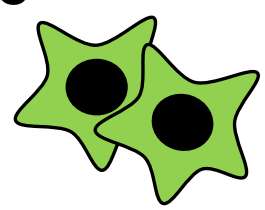

Normal

(Non neoplastic)

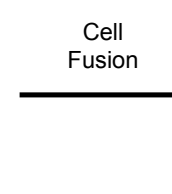

Cell

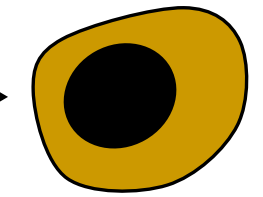

Genomic

Catastrophe

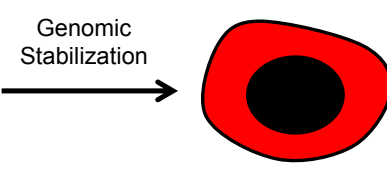

Neoplastic (Tumorigenic)
Figure 1 Molecular pathways to neoplastic transformation and tumorigenesis. A: Carcinogenesis is accepted to be a multistep process where successive molecular alterations occur over time, and each one produces some growth advantage that enables emergence of a new dominant clonal population. With accumulation of the critical number (and nature) of molecular events, neoplastic transformation occurs and a clonal population with tumorigenic potential is established. B: During neoplastic transformation, critical molecular targets are activated or inactivated through genetic or epigenetic mechanisms. Point mutations can activate or inactivate critical genes leading to neoplastic transformation. Alternatively, chromosomal alterations or epigenetic changes (like DNA hypermethylation) may contribute (along with point mutations) to neoplastic transformation. The critical number of events has not been established. C: Genomic catastrophe is a mechanism for the concurrent generation of the critical number (and nature) of molecular events over a very short period of time resulting in neoplastic transformation and tumorigenic potential in the incipient cell population. Genomic catastrophe may require a precipitating event in which an unstable tetraploid intermediate is established secondary to cell fusion or some other cellular event.
Furthermore, examination of the ploidy state of fusionderived IEC-6 cell clones over time in culture revealed karyotypic instability where near-tetraploid clones became near-diploid after time in cell culture. ${ }^{4}$ Fusion-derived IEC-6 cell clones were also heterogeneous with respect to ploidy; near-diploid clones with a modal chromosome number of 42 exhibited a wide range of chromosome numbers indicating the presence of significant subpopulations of aneuploid cells within the cell population. ${ }^{4}$ These observations combine to suggest that early after a cell fusion event, the genomes of fusion-derived clones exhibit instability that manifests as changes in ploidy, with generation of aneuploid clones, neardiploid clones that likely have aberrant karyotypes despite a near-normal chromosome number, and near-diploid clones that contain significant subpopulations of aneuploid cells. In addition to chromosomal instability, fusion-derived IEC-6 cell clones exhibited DNA damage in the form of doublestrand breaks; $35 \%$ to $42 \%$ of cells from fusion-derived clones exhibited evidence of double-strand breaks compared to only $4 \%$ to $9 \%$ of nonfused clones. ${ }^{4}$ Despite DNA damage in fusion-derived IEC- 6 cell clones, activated caspase 3 was rarely detected, indicating that DNA damage does not lead to apoptosis in these cells.

\section{Cell Fusion Leads to Neoplastic Transformation}

The results indicate that chromosomal instability (with changes in ploidy) and DNA damage (double-strand breaks) rapidly follow a cell fusion event. Zhou et $\mathrm{al}^{4}$ examined cellular growth characteristics in vitro for evidence of neoplastic changes in fusion-derived IEC-6 cell clones. A subset of fusion-derived clones (32\%) lost contact-inhibition after 12 passages in vitro, whereas $29 \%$ of fusion-derived clones acquired anchorage-independent growth capability. ${ }^{4}$ In contrast, $3 \%$ of non-fused clones lost contact inhibition, and $2 \%$ acquired anchorage-independent growth capability over the same period of time in culture. ${ }^{4}$ These results suggest that fusion-derived IEC-6 cell clones display cellular growth characteristics consistent with neoplastic transformation. However, in many cases, in vitro cellular characteristics do not accurately predict tumorigenic potential in vivo. Hence, to definitively test whether a cell fusion event can lead to neoplastic transformation, Zhou et $\mathrm{al}^{4}$ transplanted cells from a pool of fusion-derived IEC-6 cells into immunodeficient mice and tumor formation was monitored over 12 weeks. Tumors were produced in 11 of 18 host mice $(61 \%)$, whereas no tumors were produced from the transplantation of the parental IEC- 6 cells or non-fused clones. ${ }^{4}$ In addition, fusionderived IEC- 6 cell clones ( $n=9$ tested) that lost contact inhibition and acquired anchorage-independent growth potential formed tumors on transplantation with great efficiency. In contrast, fusion-derived IEC-6 cell clones that did not demonstrate cellular growth characteristics associated with neoplastic transformation in vitro failed to form tumors on transplantation into immunodeficient mice $(n=2$ clones tested). ${ }^{4}$ Of note, the karyotype of tumorigenic fusion-derived IEC-6 cell clones did not change during the process of tumorigenesis in vivo, and the tumorigenic properties of fusion-derived clones (the percentage of hosts developing tumors and tumor growth rate) were stable, suggesting that these properties were established at the time of cell fusion or rapidly following that event. ${ }^{4}$ These results combine to indicate that i) cell fusion can lead to neoplastic transformation 
in vitro, ii) pooled fusion-derived cells form tumors in vivo, iii) fusion-derived clones that exhibit growth characteristics associated with neoplastic transformation in vitro form tumors in vivo with high efficiency, and iv) tumorigenesis in vivo does not require ongoing chromosomal instability or genomic evolution.

\section{Genomic Catastrophe, A Molecular Pathway to Neoplastic Transformation}

Neoplastic transformation and tumorigenesis are well documented to be associated with genetic and epigenetic changes occurring in incipient cancer cells. It is generally accepted that these alterations accumulate over time with clonal evolution of cell populations before emergence of a clone that has tumorigenic potential (Figure 1A). The number of molecular alterations required for neoplastic transformation and tumorigenesis has been debated, and in some cases has been experimentally dissected. ${ }^{9}$ It is now accepted that most adult human cancers contain numerous genetic lesions. ${ }^{2}$ The majority of molecular models of neoplastic transformation and tumorigenesis involve multiple molecular events which occur randomly, in response to some carcinogenic insult, or as a consequence of a prior molecular event, resulting in subpopulations of cells with some growth advantage that subsequently emerge as the dominant clonal population over time. These molecular events may reflect point mutations in critical genes with oncogenic potential (which might represent activation of proto-oncogenes or inactivation of tumor suppressor genes) as depicted in Figure 1B. Alternatively, the molecular events that confer a growth advantage to specific clonal populations may reflect chromosomal alterations or epigenetic changes.

In most cancers, combinations of sequence alterations (like point mutations), chromosomal changes (like ploidy changes), and epigenetic modifications (like changes in DNA methylation) affecting positive and negative mediators of neoplastic development will drive tumorigenesis. The results generated by Zhou et $\mathrm{al}^{4}$ provide evidence for another molecular mechanism driving neoplastic transformation, genomic catastrophe (Figure 1C). The cause of genomic catastrophe is not clear, but the consequences reflect numerous molecular alterations that occur concurrently or over a short period of time leading to chromosomal instability, ploidy changes, and DNA damage. Subsequent to the period of genomic upheaval, the genomes of affected cells stabilize rapidly, in many cases assuming a chromosome number that is near-diploid, but is otherwise not normal. Zhou et $\mathrm{al}^{4}$ show clearly that some cell clones emerge from genomic catastrophe with i) the ability to proliferate in vitro, ii) cellular growth characteristics of neoplastic transformation in vitro (loss of contact inhibition, anchorageindependent growth), and iii) the capacity to form tumors in vivo. The observations of Zhou et $\mathrm{al}^{4}$ suggest strongly that genomic catastrophe can produce the required and necessary molecular alterations for neoplastic transformation and tumorigenesis in normal founder cells in the absence of selective pressures or ongoing genomic evolution.

\section{Cell Fusion Is a Model for Genomic Catastrophe}

The frequency of cell fusion events in vivo is not known, although cell fusion may occur under some circumstances such as cell injury, inflammation, and viral infection. Although the fusion of normal cells in vitro and in vivo may be a rare event, the study by Zhou et $\mathrm{al}^{4}$ shows that cell fusion between normal cells can have pathological consequences. Given the potential rarity of cell fusion events among normal cells in vivo, the relevance of the study by Zhou et $\mathrm{al}^{4}$ to the development of human cancers could be questioned. However, the cell fusion model used in this study may represent a reasonable model for other molecular and cellular events that contribute to the development of human cancers. Cell fusion between two diploid cells produces a tetraploid cell with an unstable chromosomal content which can precipitate genomic catastrophe ${ }^{10}$ with development of a stable aneuploid or near-diploid clone. Likewise, failure of cytokinesis ${ }^{11}$ or mitotic slippage ${ }^{12}$ after DNA replication results in a tetraploid cell that has stalled during cell division. Tetraploid cells resulting from these mechanisms might also be subject to genomic catastrophe and could represent the precursor cells to some cancers. Among proliferative cell populations (like skin, crypt of the colon, bone marrow, and others), failure of cytokinesis and/ or mitotic slippage may occur rarely but with some regularity. The common feature to each of these scenarios is the generation of an unstable tetraploid cellular intermediate which creates the opportunity for genomic catastrophe.

\section{Future Perspective and Remaining Questions}

Cancer is a major health issue worldwide, and some human cancers are tightly linked to specific carcinogenic exposures (such as cigarette smoking and lung cancer or sun exposure and skin cancer), familial predispositions (like hereditary colorectal cancer or breast cancer), or infectious agents (like HPV infection and cervical cancer). Other major forms of cancer are not so easily linked to carcinogenic exposures, genetic predispositions, or infectious agents. Hence, many forms of human cancer have been classified as spontaneous based on the absence of an identifiable carcinogenic agent. In most cases, spontaneous cancers derive from cells that sustain random DNA damage (related to endogenous cellular processes and reactive byproducts) or random errors during DNA replication (related to the fidelity of the DNA polymerase enzymes). In both of these examples, mutations occur very rarely because of the efficiency of the various mechanisms for cellular protection and DNA repair operating in human cells. Zhou et $\mathrm{al}^{4}$ provide evidence for a different mechanism of spontaneous neoplastic transformation. Their 
model system suggests that some spontaneous cancers could emerge from cell fusion events between normal cells, with subsequent genomic catastrophe leading to the generation of required or necessary oncogenic events that drive neoplastic transformation. We might also speculate that similar molecular events leading to neoplastic transformation may occur in other circumstances where an unstable tetraploid intermediate is generated, such as in failure of cytokinesis or mitotic slippage during normal cell division.

Although the study of Zhou et $\mathrm{al}^{4}$ provides important experimental data related to genomic catastrophe and cancer development, several important questions remain. It will be interesting to see if cell fusion events between normal human cell types (or primary cells) result in genomic catastrophe and neoplastic transformation. Likewise, it will be of interest to see if modeling failure of cytokinesis or mitotic slippage in normal human cell types (or primary cells) will result in genomic catastrophe and neoplastic transformation. These investigations can be accomplished in vitro using a similar experimental design to that described by Zhou et al. ${ }^{4}$ Beyond these questions, the specific consequences of genomic catastrophe need to be described. What gene expression changes accompany genomic catastrophe, and how do the changes in expression patterns relate to specific gene mutations or chromosomal alterations? Investigations are also needed to explore the molecular mechanisms that govern genomic catastrophe and protect fusion-derived clones from apoptosis. With additional technological development, experimental systems may emerge that will enable investigation of these challenging questions in vivo: i) How often do cell fusion events occur between normal cells in vivo? ii) Does genomic catastrophe follow cell fusion events in vivo? iii) Can failure of cytokinesis or mitotic slippage give rise to unstable tetraploid cells in vivo? iv) Does genomic catastrophe follow failure of cytokinesis or mitotic slippage in vivo?

\section{References}

1. Boveri T: [Concerning the Origin of Malignant Tumours]. German: Jena, Germany, Gustave Fischer Verlag, 1914

2. Vogelstein B, Papadopoulos N, Velculescu VE, Zhou S, Diaz LA, Kinzler KW: Cancer genome landscapes. Science 2013, 339:1546-1558

3. Hanahan D, Weinberg RA: Hallmarks of cancer: the next generation. Cell 2011, 144:646-674

4. Zhou X, Merchak K, Lee W, Grande JP, Cascalho M, Platt JL: Cell fusion connects oncogenesis with tumor evolution. Am J Pathol 2015, 185:2049-2060

5. Lazova R, Laberge GS, Duvall E, Spoelstra N, Klump V, Sznol M, Cooper D, Spritz RA, Chang JT, Pawelek JM: A melanoma brain metastasis with a donor-patient hybrid genome following bone marrow transplantation: first evidence for fusion in human cancer cells. PLoS One 2013, 8:e66731

6. Ogle BM, Cascalho M, Platt JL: Biological implications of cell fusion. Nat Rev Mol Cell Biol 2005, 6:567-575

7. Lu X, Kang Y: Cell fusion as a hidden force in tumor progression. Cancer Res 2009, 69:8536-8539

8. Quaroni A, Wands J, Trelstad RL, Isselbacher KJ: Epitheliod cell cultures from rat small intestine. Characterization by morphologic and immunologic criteria. J Cell Biol 1979, 80:248-265

9. Hahn WC, Weinberg RA: Rules for making human tumor cells [Erratum appeared in N Engl J Med 2003, 348:674]. N Engl J Med 2002, 347:1593-1603

10. Berndt B, Zanker KS, Dittmar T: Cell fusion is a potent inducer of aneuploidy and drug resistance in tumor cell/normal cell hybrids. Crit Rev Oncog 2013, 18:97-113

11. Hayashi MT, Karlseder J: DNA damage associated with mitosis and cytokinesis failure. Oncogene 2013, 32:4593-4601

12. Ganem NJ, Storchova Z, Pellman D: Tetraploidy, aneuploidy and cancer. Curr Opin Genet Dev 2007, 17:157-162 